\title{
Research on the Development of Campus Basketball in Zhanjiang Middle School in the New Era
}

\author{
Youqiang Liư ${ }^{1}$, Jingzhi Chen ${ }^{2}$ \\ ${ }^{1}$ Fujian Normal University, Fuzhou, China \\ ${ }^{2}$ Zhejiang Normal University, Jinhua, China \\ Email: 824620111@qq.com
}

How to cite this paper: Liu, Y.Q. and Chen, J.Z. (2022) Research on the Development of Campus Basketball in Zhanjiang Middle School in the New Era. Open Access Library Journal, 9: e8288.

https://doi.org/10.4236/oalib.1108288

Received: December 10, 2021

Accepted: January 7, 2022

Published: January 10, 2022

Copyright $\odot 2022$ by author(s) and Open Access Library Inc.

This work is licensed under the Creative Commons Attribution International License (CC BY 4.0).

http://creativecommons.org/licenses/by/4.0/ (c) (i) Open Access

\begin{abstract}
The development of middle school campus basketball has always been an important issue that experts are studying. There are many scholars in our country who have published different views and articles in this field from different angles. How to improve Zhanjiang middle school campus basketball is the purpose of this paper. This paper investigates several primary and secondary schools in Zhanjiang City by means of literature, field investigation and expert interview. Summarizes the Zhanjiang middle school campus basketball in the development of the process of the existence of a series of problems. From the school, teachers, students, competition system, sponsors and other five perspectives to improve the efficiency of campus basketball, Zhanjiang middle school campus basketball development provides a theoretical basis and practical basis for the development of campus sports, to help the development of campus sports
\end{abstract}

\section{Subject Areas}

Physical Education

\section{Keywords}

Zhanjiang, School Basketball, Physical Exercise

\section{Introduction}

General Secretary Xi Jinping made a clear instruction at the National Education Conference in 2018: "We should establish the education concept of health first, provide all physical education classes, and help students enjoy physical exercise, 
enhance their physical fitness, improve their personality, and exercise their willpower" [1]. The important position of physical education in school education is emphasized from the strategic height of commanding the whole situation. It is emphasized that in school education, cultural classes should not occupy the time of physical education so that students can grow up in physical exercise. The physical quality of students in primary and secondary schools is in a period of rapid development. As a comprehensive project, basketball requires high physical qualities such as speed, strength, absolute explosive power, jumping, endurance, core strength and flexibility. Students can develop their physical qualities in all aspects while participating in basketball, so it has a high value of exercise. The development of campus basketball undoubtedly lays a good foundation for the development of competitive sports in China. Enrich the reserve pool of high-level basketball players in our country. The Construction of campus basketball in the United States is very good, from the development of AAU (American basketball players), AUU (American Amateur Sports League) and NSAA (National High school league) various events, and then to the support of the management system, the campus basketball atmosphere in the United States is very active [2]. At the national level, the development of NSAA has provided excellent seed players for the American basketball career and greatly aroused people's enthusiasm for sports careers. At the local level, various state-city -district level leagues are actively carried out. It can not only mobilize the local financial income, but also spread the atmosphere of basketball to various regions, make friends with the ball, and gather the world's talents. At the school level, students are encouraged to actively participate in basketball, scholarships of different levels are set to improve students' enthusiasm, and open days of colleges and universities are held regularly to let more athletes into colleges and universities social level. Through the star effect of basketball superstar, people from all walks of life are motivated to contact basketball, love basketball, enjoy the fun in the process of sports, and improve the happiness of students. In contrast, the development of campus basketball in Zhanjiang City is not paid attention to by schools, parents and society. Many parents put their children's academic performance in the first place and ignore the good benefits of physical exercise for students' physical and intellectual development, which leads to the stagnation of campus basketball in Zhanjiang City.

The topic of how to develop basketball in middle school has been concerned by experts and scholars, and many scholars in our country have expressed different opinions on it. In value Appeal and Strategy of Campus Basketball Integrated Curriculum System Construction in 2021, Li Yuanguang mentioned the advanced concept of "vertical connection, horizontal consistency, internal unity and form unity", and took basketball competition as a breakthrough point to cultivate students' interests and lifelong sports awareness [3]. Both Gao Zhi and Ren Zeming mentioned how to improve the development of Campus basketball in China in 2019 "Dynamic Mechanism Construction of The Development of 
Campus Basketball in China" [4] and 2020 "Research on the Development of Campus Basketball in China in the New Era" [5]. However, the existing research has not really improved the current situation of campus basketball in Zhanjiang City; there is no substantive solution to the problems of campus basketball in Zhanjiang City. Therefore, this paper will study how to optimize Zhanjiang campus basketball. Through the three levels of school, parents and society, the initiative of teachers, students and social people for basketball will be improved, and people's love for campus basketball will be reawakened.

\section{The Current Situation of Campus Basketball in Zhanjiang Middle Schools}

Zhanjiang city is located in Guangdong Province, located in the southern tip of the motherland, the four seasons like spring climate has cultivated a large number of excellent athletes, including Quan Hongchan (national diving team athletes, Gold medalist of 10-meter platform diving in Tokyo Olympic Games and National Games), Jin Guangzhu (the first champion of CUBA in 2016), Roger (CUBA player, Cubist and runner-up of national Slam Dunk Contest in 2010) are all outstanding representatives). In addition, several key high schools in Zhanjiang also have characteristic schools for traditional basketball projects. Taking Zhanjiang No. 1 middle school and Suixi No. 1 middle school as an example, some students get the certificate of basketball secondary player by participating in the college games every year, which is helpful for taking the single recruit route in the future, but most students are not able to participate and do not realize the popularization well. Because of the limited number of places a school can allocate each year, each school uses the school team training system. In the school, students who can join the school team training have a certain level of basketball, and finally, have to be screened out, and only a few players can stay and participate in the competition. For students who love basketball, enthusiasm has a certain degree of blow. In the process of developing campus basketball, Zhanjiang Basketball Association (ZJBA) is also developing branches in various regions. Among them, Suixi Basketball Association was formally established in Suixi Gymnasium on August 15, 2021 with 30 members; Mazhang Basketball Association was formally established in BHOLIDAY Inn Zhanjiang on December 18, 2020 with 19 members; Lianjiang Basketball Association was formally established in Kaiden International Hotel on August 29, 2019 with 25 members. All the actions to make more love the cause of basketball people to join, committed to the Zhanjiang City basketball cause carry forward.

\section{There Are Still Drawbacks in the Development Process of Campus Basketball in Zhanjiang}

\subsection{The School Curriculum Design Is Unreasonable}

Whether the design of PE class is reasonable or not directly affects students' learning efficiency in the whole class, and the reasonable arrangement of PE cur- 
riculum content directly determines students' interest in this aspect. The setting of basketball curriculum content will have a significant impact on students' learning of basketball skills. Only some schools add basketball skills and tactics in basketball class [6]. Through visiting Zhanjiang No. 1 Middle School, Zhanjiang No. 2 Middle School, Zhanjiang No. 4 Middle School, Zhanjiang Peicai Middle School, Suixi County No. 1 Middle School, Suixi Dacheng Middle School and other middle schools, and communicating with the sports section leaders and sports teachers of the schools. At present the development of Zhanjiang middle school campus basketball is generally in the form of class, in class, the teacher imparting knowledge, students will blindly follow, in the process is easy to form the teaching of the non-computer majors, most of the time are students themselves in the play, students can apply the knowledge in real combat is very little, few arrange basketball technique teaching in the classroom. Students lack practical practice, through practical practice, the knowledge learned in class will be flexible application. The cooperation between players and the confrontation between opponents can improve students' core strength and comprehensive basketball ability, and then improve the enthusiasm of the class.

\subsection{The Qualifications of PE Teachers Cannot Keep Up with the Changes of the Times}

With the increase of age, every physical education teacher and teacher team cannot avoid the weakening of human body function. Physical education is a subject with high requirements on physical strength and sports skills. Therefore, the rational allocation of teacher age structure will affect physical education teaching in schools [7]. During the $14^{\text {th }}$ five-year Period, teachers were required to carry out quality-oriented education and promote students' all-around development in morality, intelligence, physique and aesthetics. Among many physical education teachers, most of them are undergraduate or even junior college graduates, and there are very few basketball teachers. It is generally advocated that physical education teachers should take charge of everything. It is not particularly prominent in a certain sport, and cannot be corrected in time for some deep-seated mistakes easily made, so as to give students better professional guidance.

\subsection{Students Do Not Have Enough Knowledge of Basketball to Arouse Their Interest in Basketball}

Students' cognition of basketball may be seen in a certain game or a certain scene on TV. In the construction of campus basketball, the improvement of students' cognitive ability is the top priority. Some students have little knowledge about basketball, do not know the basic rules of basketball, and do not devote themselves to basketball. Such a vicious cycle, and eventually lead to no sense of basketball. The fundamental problem is that they do not understand the benefits of playing basketball. In addition, unreasonable classroom Settings and individual differences among students eventually lead to students' indifference and re- 
sistance to basketball.

\subsection{Parents Blindly Ask Their Children to Improve Their Academic Performance and Ignore Physical Exercise}

In school days, parents only pay attention to their children's academic performance and remain neutral to physical exercise. Some parents even directly deny their children's exercise, thinking that exercise is a waste of time and it is better to spare more time to study. As everyone knows, students are in a critical period of growth and development, and participating in physical exercise plays a positive role in promoting children's psychological, physiological and physical aspects. Basketball, as a behavior conducive to the overall development of physical and mental health, plays an irreplaceable role in promoting students' overall development [8]. Only in the case of reasonable exercise, students' physical fitness will be further improved. Physical and mental development will also be better.

\subsection{The Competition System Lacks Overall Planning}

Zhanjiang middle school campus basketball sports in various schools have different degrees of development. Most of them are class competitions. Friendlies between friends are common and there is no system to support them. There is no effective linkage between the upper and lower grades. There is no reasonable arrangement from top to bottom. Compared with the competition held by schools in developed areas, the form of the activity is relatively simple, only simple in-class competition. The small size of the school, the small number of students, and the lack of management of the facilities all year round limit the scale and form of the competition [7]. Most of the referees are directed and played by students themselves. Since students have not received professional training and do not have a thorough understanding of the rules of refereeing, there will often be some misjudgments or omissions, which will deal a big blow to the confidence of the players on the court, and thus affect students' interest in basketball.

\subsection{Investment of Advertising Sponsors}

Most of the funds of Zhanjiang Basketball League are provided by clubs, and the government has not realized the last resort and expanded the publicity. Add to that the lack of advertising and sponsorship. Lead to problems in the connection of each link. Grassroots seeds have not been effectively mined, there is no fair and transparent transmission channel, the upper demand and the lower supply cannot match. In addition, due to the limitation of geographical location, many large sports companies have not settled in Zhanjiang, which to some extent affects the development of sports undertakings in Zhanjiang.

\section{Discussion and Suggestions}

\subsection{Increase the Investment of School Sports Funds}

The biggest difference between PE class and other theoretical classes is that the 
body has to bear a certain amount of load, and the diversity of teaching organizations requires the classroom to have a certain degree of dependence on the site equipment. Since most physical exercises are conducted outdoors, the site equipment plays a crucial role as a medium. Good field equipment can reduce the risk of sports, so a physical education on the field equipment is relatively high requirements. The effect of physical education is largely related to the school's equipment, so the school should increase the investment in sports funds to ensure that there is enough equipment for the full use of physical education teachers in teaching.

\subsection{Enhance Students' Cognition of Basketball and Improve Teaching Methods}

To take the student as the main body in the process of teaching activities, teachers as the leading form, teachers should fully according to students' physical and psychological conditions, according to the teaching material content, to the difficulty of the achieved standard, the focus of the show, as well as the interpretation of the way of demonstration and so on, all want to accord with students' physical and mental development of the current level, give full play to students' subjective initiative, Organize rich and colorful teaching contents and choose diverse teaching methods to improve students' enthusiasm for learning. Adopt more question-based and situational teaching methods in the teaching process to guide students to think positively about what they want to learn and promote the combination of theory and practice. More intuitive teaching method, through video games, improves students' cognition of basketball, expand domestic demand.

\subsection{Improve the Teaching Enthusiasm of Physical Education Teachers, Improve the Work of Teachers}

Physical education teacher is the leader of physical education teaching, but also the guide and enlightenment of students. The enthusiasm and investment of physical education teacher in class indirectly affects the effect of students learning new knowledge. The most direct way is to improve the physical education teachers' working treatment, which is to introduce higher talents through high salary and feedback teaching through students' evaluation. To promote teachers to realize personal value, love work, so as to improve the enthusiasm of teaching, improve the quality of teaching.

\subsection{Improve the Social Attention to Campus Basketball}

Improve the management system of campus basketball, strive to combine campus basketball with social basketball, regularly hold school league within the region, can also hold inter-regional league. Schools with conditions can participate in outside social leagues. Let more people participate in campus basketball, feel the charm brought by basketball, improve the influence of basketball on society. 
Enhance zhanjiang campus basketball atmosphere, let more parents know campus basketball.

\subsection{To Ensure That the Government Is in the Back Pocket, and Vigorously Develop Sports Undertakings}

Through the government to issue a series of policies to benefit the people, improve the enthusiasm of sports clubs to participate in Zhanjiang campus basketball construction, regularly hold large-scale basketball competitions every year, from the city-district-county-town-village overall planning. Generous prize money will be given to the winners and runners-up. Students will be given athlete certificates and their scores will be included in the gaokao scoring criteria. Truly realize the national exercise, national exercise, and national fitness.

\subsection{Reform the System for Connecting Children, Primary Schools, Middle Schools and Universities}

From kindergarten to university, reform will be deepened. Realize reverse design teaching from university to children. Make clear the tasks and objectives of each stage and link up each stage effectively. In the kindergarten stage, give children the right direction, spread the seeds of basketball; Primary school, focus on cultivating children's interest in basketball, so that children have good enlightenment for basketball; In junior and senior high schools, students' physical quality is mainly cultivated. Through basketball, students' strength, speed, flexibility, agility, coordination, endurance and other aspects of physical quality are effectively improved and lifelong sports awareness is established. College stage, through professional training, high-intensity competition and professional basketball skills, continue to carry out technical and tactical training, hone cooperation, enhance the strength of physical confrontation and train excellent basketball reserve talents for the country.

\subsection{Encourage In-Service Teachers to Improve Their Educational Background}

For a teacher, continuous improvement of learning and reflection is the best way to improve the teaching level of teachers. It is necessary to optimize the in-service training of physical education teachers and improve the channels for academic qualifications. So that PE teachers can constantly improve their cultural level and teaching skills in the teaching process. Contact with the latest teaching ideas, learn cutting-edge teaching methods, form the concept of lifelong learning, and finally feedback teaching practice to serve the student community.

\section{Conclusion}

The development of campus basketball in Zhanjiang middle school needs to be improved. This paper analyzes the shortcomings of campus basketball in Zhanjiang City from five different perspectives, including schools, teachers, students, 
competition system and sponsors, and puts forward corresponding suggestions for improvement. It is necessary to increase the investment of school sports funds, enhance students' cognition of basketball, improve teaching methods, improve the social attention to campus basketball, realize the government's support, vigorously develop sports, and reform the link system of every stage from kindergarten to primary school to middle school to college. At the national level, we should vigorously advocate campus basketball and issue a series of additional score policies to stimulate students' enthusiasm for basketball. At the local level, we actively respond to the call of the state, build a good competition platform, and promote the development of Zhanjiang campus basketball with a fair, just and open attitude. At the school level, the first productive force is to introduce talents. Improve students' interest in basketball through diversified curriculum design. Regular inter-school tournaments are held, mainly by physical education teachers, as basketball sanctions. Hold more basketball knowledge competitions to enrich students' spare time life. The only thing missing is the lack of laboratory equipment. There is no experimental demonstration to analyze the influence of basketball on the physical and mental development of primary and secondary school students of all ages. There is no data summary analysis, students participating in basketball has a positive effect on the body, height, intelligence development brought benefits.

\section{Conflicts of Interest}

The authors declare no conflicts of interest.

\section{References}

[1] Ji, L. (2020) Improving Students' Physical and Mental Health Is the Fundamental and Direction of the Development of School Physical Education in China-To Study and Implement the Important Spirit of General Secretary Xi Jinping's Speech at the National Education Conference. Journal of Jishou University (Social Science Edition), 9, 28-37.

[2] Liu, Y. (2017) Exploration and Enlightenment: Research on the Development of American Campus Basketball Culture. Journal of Shenyang Institute of Physical Education, 36, 47-52.

[3] Li, Y.C. and Guo, Y.B. (2021) Our Campus Basketball Value Aim and Strategy of the Construction of the Integrated Curriculum. Journal of Sport, 28, 90-96.

[4] Gao, Z. and Xu, Q. (2019) Chinese Youth Campus Basketball Movement Development Dynamic Mechanism to Build. Journal of Wuhan Sports College, 2019, 82-87.

[5] Ren, Z.M. and Chen, H. (2020) Research on Campus Basketball Development in China in the New Era. Sports Culture Guide, 2020, 105-110.

[6] Liu, Y.J. (2020) Henan Province High School Campus Basketball Activity Promotion Strategy Research. Master Thesis, Henan University, Kaifeng.

[7] Wang, J.K. (2020) Research on the Current Situation and Countermeasures of Campus Basketball Development in Huizhou Senior Middle Schools. Master Thesis, Guangxi Normal University, Guilin.

[8] Zhu, W., Li, G.X. and Ye, X.H. (2021) Research on the Promoting Effect of Sports on College Students' Mental Health. Sport \& Style, No. 10, 20-21. (In Chinese) 